\title{
Rosette-Forming Glioneuronal Tumor of the Fourth Ventricle
}

National Cancer Institute

\section{Source}

National Cancer Institute. Rosette-Forming Glioneuronal Tumor of the Fourth Ventricle. NCI Thesaurus. Code C67559.

A central nervous system neoplasm arising from the fourth ventricle. It is characterized by the presence of neurocytes forming pseudorosettes and astrocytes which contain Rosenthal fibers. Cytologic atypia is minimal. 